DOI: $\underline{\text { http://dx.doi.org/10.35381/r.k.v4i8.275 }}$

\title{
Transferencia de conocimiento como estrategia gerencial del sector farmacéutico en Colombia
}

\section{Transfer of knowledge as a management strategy for the pharmaceutical sector in Colombia}

\author{
Elkin Baleta Araujo \\ lebael02@gmail.com \\ Universidad Popular del Cesar \\ Colombia \\ https://orcid.org/0000-0002-2942-7281
}

Recibido: 30 de enero del 2019

Aprobado: 07 de abril del 2019

\section{RESUMEN}

El objetivo general del presente estudio fue analizar la transferencia del conocimiento como estrategia gerencial del sector farmacéutico en Colombia, fundamentado en autores Krogh (2000); Nonaka y Takeuchi, (1999), entre otros. Enmarcado bajo un paradigma racional, el diseño se fundamentó en un trabajo bibliográfico. En las organizaciones es necesario contar con el conocimiento para ser aprovechado idóneamente aplicado a través de un servicio eficiente para ser competitivos en la sociedad cambiante y transformadora. En tanto las organizaciones del sector farmacéutico, permiten resaltar la interactividad e inspiración del recurso humano para el desarrollo de los objetivos asignados, con la finalidad de construir metas propiciando cambios en los diferentes procesos efectuados en la institución. La misión, visión, valores, objetivos y metas son características que condicionan los resultados en los niveles estratégicos, debido a la relevancia hacia la excelencia en el desarrollo de condiciones para alcanzar eficiencia y eficacia en los servicios prestados.

Descriptores: Transferencia del conocimiento; estrategia gerencial; sector farmacéutico; analítico; bibliográfico. 


\section{ABSTRACT}

The general objective of the present study was to analyze the transfer of knowledge as a management strategy of the pharmaceutical sector in Colombia, based on authors Krogh (2000); Nonaka and Takeuchi, (1999), among others. Framed under a rational paradigm, the design was based on a bibliographic work. In organizations it is necessary to have the knowledge to be used appropriately applied through an efficient service to be competitive in the changing and transformative society. In as much the organizations of the pharmaceutical sector, allow to emphasize the interactivity and inspiration of the human resource for the development of the assigned objectives, with the purpose of constructing goals propitiating changes in the different processes carried out in the institution. The mission, vision, values, objectives and goals are characteristics that condition the results at the strategic levels, due to the relevance towards excellence in the development of conditions to achieve efficiency and effectiveness in the services provided.

Descriptors: Knowledge transfer; managerial strategy; pharmaceutical sector; analytical; bibliographic.

\section{INTRODUCCIÓN}

El mundo contemporáneo asiste a un genuino cambio de época, el cual se evidencia en el desarrollo social, científico y tecnológico de las sociedades que buscan el progreso, obligadas a incorporar nuevas formas de vida y de comunicación. De esta forma, en las organizaciones es necesario contar con el conocimiento ya que puede ser aprovechado idóneamente al ser aplicado a través de un servicio eficiente que permita tomar en cuenta todos los elementos para ser competitivos en la sociedad cambiante y transformadora que actualmente se desarrolla.

De tal manera, que la transferencia de conocimiento representa un mecanismo fundamental para el logro de cualquier actividad empresarial, ya que se conjugan los saberes de quienes hacen posible alcanzar determinados objetivos y metas. Hoy en día existe un cambio en el modo del desarrollo organizacional, en el que se presume al conocimiento como el mayor valor agregado de los bienes, servicios sociales, culturales, como económicos que se generan, sobre todo en América Latina. 
De lo anterior, es importante referir que para la transferencia del conocimiento sea viable es un compromiso de todos, es importante que exista una asociación entre los factores tanto gerenciales, como individuales, enfocada en conductas dirigidas al éxito empresarial; el sector farmacéutico está dedicado a la fabricación, preparación y comercialización de productos químicos medicinales para el tratamiento, también la prevención de las enfermedades, hace parte fundamental de la generación del nuevo conocimiento, el progreso tecnológico y la innovación como factores determinantes en el crecimiento de esta economía en Colombia.

Por otra parte, los gerentes se convierten en intermediarios gestionando la dinámica interna y externa de la organización en cuanto al progreso de aprendizaje y la estrategia gerencial, logrando; su principal compromiso, dirigido a articular entre la adquisición y generación de conocimientos en el entorno donde se encuentra. En este sentido, es importante resaltar que los saberes en el sector farmacéutico, están intrínsecamente relacionados con la gerencia estratégica, ya que ésta fundamenta todo el sistema de la ventaja competitiva, marcando una visión compartida, la cual crea una comunidad de intereses que impulsa y la coloca a la expectativa de nuevas oportunidades y retos.

\section{FUNDAMENTACIÓN TEÓRICA}

Para el desarrollo de los fundamentos teóricos de esta investigación, se consideró conveniente presentar diferentes definiciones de autores desde su perspectiva científica a partir de las discusiones y propuestas que generan los postulados, que de alguna manera se relacionan con la investigación y por consecuencia representan aportes para el estudio. De tal manera, que al especificar aquellos aportes que abren perspectivas a discusiones de reafirmación teórica, se permiten explicar de manera detallada, concisa y profunda todo el proceso de investigación:

\section{Transferencia de conocimiento}


La generación del nuevo conocimiento, el progreso tecnológico y la innovación son factores determinantes en el crecimiento de una economía, hoy en día experimentan procesos que tienden a formar sus sociedades e industrias en economías basadas en el conocimiento. Para, Gibson, Ivancevich, Donnelly y Konopaske (2015), afirman que el transferencia de conocimiento es el resultado de un entendimiento común, en pocas palabras, la persona que desea comunicar algo o transmitir información, generalmente, busca establecer una condición común con el receptor, así como, la comprensión a través del uso de símbolos comunes que les permitan entenderse o llegar a acuerdos que beneficien a ambas partes.

Asimismo, Ordóñez (2011), tomado de Nonaka y Takeuchi (1999), lo definen como la capacidad de la empresa para crear conocimiento nuevo, diseminarlo en la organización e incorporarlo en productos, servicios y sistemas. Desde diversos ámbitos ha surgido un enorme interés en la gestión del conocimiento, pues este activo intangible es una fuente indiscutible de valor organizativo.

En este mismo orden, Cohen y Levinthal, (1990); Krogh (2000); Nonaka y Takeuchi, (1999); Lam, (1999), mencionan a la transferencia de conocimiento como un proceso de interacción social orientado hacia la producción y circulación de conocimiento que genera externalidades de aprendizaje. Este proceso interactivo es interno y externo a la organización, combina distintas capacidades y recursos organizacionales, constituye microcomunidades de conocimiento $y$ articula diferentes tipos $y$ formas de conocimientos.

En otras palabras, el conocimiento abunda en las organizaciones, pero su existencia no garantiza su uso; la empresa debe orientarse a la generación de conocimiento, en el sentido de crear valor o incrementarlo en todo caso y darle utilidad al conocimiento transferido en los procesos y las actividades diarias de una empresa.

\section{Modalidad en la transferencia de conocimiento}


La modalidad en el proceso de la transferencia de conocimiento, de acuerdo a Testar (2012), se puede asimilar desde la cadena de valor que puede ser endógena, exógena y comunidades de conocimiento, lo cual puede permitir transitar desde la investigación, la I+D, que se lleva a cabo en el entorno público, hasta su transformación en nuevos o mejorados productos y servicios que las empresas trasladarán al mercado, es decir a los ciudadanos y potenciales clientes, a través de la innovación. El análisis de las modalidades de transferencia de conocimiento se puede abordar de múltiples formas, pero en este caso es la transferencia entre investigación pública y empresa, una primera clasificación puede realizarse considerando donde reside la fuerza motriz del proceso.

En este sentido, González (2011), menciona que las empresas pueden crear innovaciones tecnológicas deben disponer de las tecnologías adecuadas para ello, y éstas pueden obtenerse a partir de dos fuentes que pueden ser complementarias: Fuentes internas: departamentos propios de investigación y desarrollo tecnológico $(\mathrm{I}+\mathrm{D})$. Fuentes externas: colaboración con proveedores y socios externos ajenos a la empresa para la adquisición de la tecnología cuando ya está disponible o el desarrollo de la misma en caso contrario y de este modo lograr la transferencia de conocimiento como ventaja competitiva.

El autor también afirma, que conforme la empresa se pondere más por el extremo de la creación de tecnología propia tenderá hacia un mayor interés en liderazgo tecnológico, una mayor incursión en riesgos técnicos y una mayor utilización de recursos (humanos, técnicos y económicos). La transferencia de tecnología y conocimiento constituye en último término una de las fuentes de la innovación de las empresas. Por ello, tan importante como la generación del conocimiento y la tarea de la unidad de interfaz, es el entorno en que se desarrollan. Es decir, la existencia o no de un contexto innovador de empresas capaces de identificar en el ámbito de la I+D pública resultados y capacidades de su interés que puedan ser incorporados a sus procesos productivos, a través del proceso de innovación. 


\section{Mecanismos para la transferencia de conocimiento}

Para establecer los mecanismos para la transferencia del conocimiento cada sistema organizacional tiene sus modos para establecer estos mecanismos de transferencia de aprendizaje que permitan y el movimiento y captar el conocimiento necesario para su ejercicio, o a la comunidad.

De esta forma, Rodríguez (2009), tomado de (Dyer y Nobeoka, 2000) menciona que las directivas explícitas pueden ser mecanismos más efectivos cuando se acompañan con otros mecanismos más sociales, es decir, de interacción directa entre las personas, denominados rutinas inter-organizacionales. Las rutinas son patrones regulares de interacción, diseñados para facilitar la transferencia de conocimiento especializado tanto explícito como tácito (modelos mentales, creencias y perspectivas) entre los miembros de la organización, requieren contacto personal para intercambiar el conocimiento.

Por otro lado, de acuerdo con Dawson (2010) este mecanismo para la transferencia de conocimiento es el proceso mediante el cual el conocimiento propiedad de una persona, grupo o entidad emisora es comunicado a otra persona, grupo o entidad receptora que tiene una base común o conocimiento similar al del emisor. Fija su posición refiriendo que existe una relación entre dos y son muy variados los mecanismos que pueden ser usados para transferir conocimiento, pudiendo clasificarse entre directivas explícitas, rutinas explícitas y rutinas tácitas.

De acuerdo, a Vega (2015), refiere que de acuerdo a los criterios que determinan los mecanismos de transferencia del conocimiento en cuanto a quién es el receptor propuesto y la naturaleza de la tarea, pues será el tipo de conocimiento que habrá de transferirse.

Este mismo autor asevera que las organizaciones deben reinventar y actualizar continuamente su conocimiento común; refiere que de acuerdo a los criterios que determinan los mecanismos de transferencia del conocimiento en cuanto a quién es el 
receptor propuesto y la naturaleza de la tarea, pues será el tipo de conocimiento que habrá de transferirse el autor menciona cinco mecanismos de transferencia del conocimiento en donde cada una de las cuales requiere de diferentes elementos de diseño para hacer que la transferencia de conocimiento resulte satisfactoria, en serie, cercana, lejana, estratégica y experta.

\section{Estrategia gerencial}

Davis y Newstron (2008), exponen que la estrategia gerencial en general no es más que el proceso de dirigir las actividades laborales de los miembros de un grupo y de influir en ellas, la estrategia gerencial involucra a otras personas; a los empleados 0 seguidores. La estrategia gerencial es definida por Utrilla (2009) es como un plan destinado a dirigir o guiar todas las acciones hacia un fin específico o meta, en la cual se debe incluir todas las herramientas, elementos y decisiones necesarios oportunamente, teniendo en cuenta cambios inesperados del mercado.

Ahora bien, Serna (2006), argumenta que en el campo de la estrategia gerencial, es importante implantar en la organización estrategias como: los principios corporativos relacionados al mapa estratégico (misión, visión, objetivos, valores y metas) orientados a tomar decisiones relevantes para el buen funcionamiento de la empresa. De igual manera, argumenta que existen otras estrategias como la investigación (buscar información), tomar decisiones, comunicar, liderar.

\section{Competencias de la estrategia gerencial}

Reyes (2011), refiere que existen tres componentes individuales que permiten entender las competencias dentro de la estrategia gerencial, en estas se encuentras las técnicas, las específicas y las genéricas. De las Salas y Bozo (2014) refieren que las competencias de la estrategia gerencial representan un conjunto de comportamientos, actitudes, habilidades y valores que una persona posee y que, al ser aplicadas a cualquier situación posibilitan la eficacia en una actividad determinada. 
Puede mencionarse, por ejemplo: la comunicación oral y escrita, análisis de problemas, delegación, creatividad, entre otras, las cuales hacen referencia a las cualidades propias de un individuo y la capacidad para efectuarlas correctamente en un contexto determinado.

Según Barroso (2009), las competencias gerenciales como comportamientos y destrezas visibles que la persona aporta, para cumplir con sus responsabilidades de manera eficaz y satisfactoria. Destaca las competencias genéricas como cualidades o atributos directamente relacionados con el desempeño en grupos de empleos determinados y afines, no son generalizables entre las diferentes áreas o niveles organizacionales.

Por otra parte, considera las competencias definiéndolas como un grupo de competencias mínimas y comunes a los diferentes sectores laborales, áreas o niveles de gestión. Son los cimentos que fundamentan el desempeño adecuado y el aprendizaje de transferir a la cotidianidad información, conocimientos, aptitudes y habilidades desarrolladas.

\section{METODOLOGÍA}

Para el desarrollo del estudio se consideró pertinente analizar transferencia de conocimiento como estrategia gerencial del sector farmacéutico en Colombia, desde las dimensiones filosóficas, epistemológicas y metodológicas, bajo el enfoque positivista, por partir éste de las realidades, cuya naturaleza, estructura característica sólo pueden ser captadas desde el marco de referencia del sujeto que las vive y las experimenta, en un momento y de un espacio determinado. De igual manera, se hace explicación del enfoque, paradigma o posición epistemológica, la cual representan las diversas expectativas a las que puede optar el investigador en el desarrollo de las investigaciones científicas, que permiten identificar la perspectiva, sistemas, postulados y métodos, que soportan las mismas. 
Es de suma importancia analizar la corriente filosófica en el cual la investigación se construye el fundamento epistemológico surge de la estructuración de periodos históricos, políticos, económicos, geográficos, valores, costumbres y estructura social, entre otros, que se relacionan sistemáticamente para crear un mundo de vida en el que se desenvuelve el hombre y en donde exista, una práctica existencial, (forma de ser 0 de estar siendo).

Sobre la base de las ideas expuestas se deduce, que toda investigación debe definir su paradigma a partir del marco epistemológico, ontológico y metodológico que le permita generar nuevos conocimientos, los cuales serán útiles para la sociedad. En tal sentido para responder al posicionamiento epistemológico se considera que la presente investigación se identifica con el enfoque positivista, el cual para Hurtado (2010), concede la primacía a los hechos antes que a las ideas, a las ciencias experimentales antes que a las prácticas y las leyes físicas antes que los postulados de la filosofía.

Palella y Martins (2006), exponen que el positivismo no es más que el sentido común generalizado y sistematizado, sus principios fundamentales son: el rechazo a la metafísica y a todas las preposiciones desvinculadas de hechos constatados, el rechazo a los juicios de valor en cuanto no se apoyen en certezas y leyes científicas, así como la aceptación del empirismo como único medio para llevar a cabo observaciones sistemáticas y ciertas con el fin de deducir conclusiones válidas.

Así mismo, la investigación se ubicó bajo un enfoque cuantitativo, entendiendo este como aquel en el que se recogen y analizan datos cuantitativos sobre las variables, estudiando su relación o asociación. Al respecto para Hernández y Fernández y Baptista. (2014), definen este enfoque como aquel que utiliza la recolección y el análisis de datos para dar respuestas a las preguntas de investigación, probar hipótesis establecidas previamente y confía en la medición numérica, en el uso de la estadística para establecer con exactitud patrones de comportamiento de una población.

En el mismo sentido, se expone el tipo de la investigación el cual establece los pasos a seguir del estudio, técnicas y métodos que pueden ser utilizados en la misma, según 
Palella y Martins (2006), hacen referencia a la clase de estudio que se pretende realizar, orienta sobre la finalidad general del estudio y sobre la manera de recoger la información o datos necesarios.

De esa forma, se encontró que este estudio tuvo como objetivo general analizar la transferencia de conocimiento como estrategia gerencial del sector farmacéutico en Colombia, por lo tanto se considera analítico-descriptivo y, ante lo cual para Hurtado (2010), señala que la investigación analítica tiene como objetivo analizar un evento y comprenderlo en términos de sus aspectos menos evidentes incluyendo tanto el análisis como la síntesis. Es decir, implica la interpretación de lo analizado en función de criterios descritos, considerando los objetivos en estudio.

En consideración a lo anterior, plantea Hernández, Fernández y Baptista (2014), que los estudios descriptivos pretenden medir o recoger información de manera independiente o conjunta sobre los conceptos o variables a las que se refieren, esto es, su objetivo no es identificar como se relacionan las variables medidas, en este caso en particular a la trasferencia de conocimiento y la estrategia gerencial.

En relación al diseño de la investigación, Hernández y otros. (2014), va enmarcado en un plan referido a dar respuestas a interrogantes realizadas en una investigación; señala que debe ser dirigido a alcanzar los objetivos en el estudio, brindándoles respuesta a las interrogantes planteadas. De allí, que los autores mencionados anteriormente, plantean que la investigación no experimental es observar fenómenos tal y como se dan en su contexto natural, para después analizarlo.

Siguiendo el orden de las ideas expuestas, Chávez (2010), afirma que un diseño de investigación transaccional o transversal, es aquella en la cual las variables son estudiadas solo una vez y sin tomar en cuenta a la evolución de tiempo. De allí, que en éstos diseños lo que se pretende es medir y analizar (enfoque cuantitativo) o evalúa analiza (enfoque cualitativo) es la asociación entre categorías, conceptos, objetos o variables en un tiempo determinado, que vienen a ser las razones por las que se manifiesta una categoría, una variable, un suceso o concepto. 
Desde esta premisa se consideró de campo, ya que los datos se obtuvieron directamente de la población que es objeto de estudio. Para Hurtado (2010, p. 230), este diseño en una investigación; "Ileva como propósito describir un evento obteniendo los datos de fuentes vivas o directas, en su ambiente natural; es decir, en el contexto habitual al cual ellas pertenecen, sin introducir modificaciones de ningún tipo o dicho contexto".

Sobre la base de las ideas expuestas anteriormente, el diseño de la presente investigación fue no experimental, bajo la modalidad de campo, transeccional o transversal, se observó los fenómenos tal y como sucedieron en la realidad, no se constituyó ninguna situación ni se provocó ningún cambio intencionalmente por el investigador, se realizó la medición de las variable una sola vez, en un tiempo único, a fin de describirlas y analizar su comportamiento.

En toda investigación es importante definir la población que será a objeto para el estudio así como, el universo o espacio donde se lleva a cabo la misma, ya que, constituyen las unidades de análisis e información. Partiendo de esto, Arias (2012, p. 22), define la población como "el conjunto para el cual serán válidas las conclusiones que se obtengan: a los elementos o unidades (personas, instituciones o cosas) involucradas en la investigación". De allí, que el universo estuvo conformado por las (06) empresa principales del sector farmacéutico en Colombia, quedando como muestra intencional tres (03) tres de ellas, Departamento del César, Colombia, por el fácil acceso a la información y ubicación geográfica.

El éxito de una investigación de acuerdo a las técnicas seleccionadas para la recolección de información, así como en la idoneidad de los instrumentos utilizados para tal fin. En este orden de ideas, Arias (2012, p. 65), quien define técnica como "el procedimiento o forma particular de obtener datos o información. En este orden de ideas, la recolección de la información se realizó con base en la técnica de la encuesta y como instrumento un cuestionario. 
En este contexto, Briones (1990, p. 49), citado por: Hernández y otros (2014), quienes señalan que de manera general se puede definir la encuesta social como un conjunto de técnicas destinadas a recoger, procesar y analizar informaciones que se dan en unidades o en personas de un colectivo determinado.

Por lo tanto, se utilizó como instrumento tipo cuestionario, definido por Hernández, y otros (2014, p. 310$)$, quienes describe que "consiste en un conjunto de preguntas con respecto a una o más variables a medir" los cuestionarios pueden contener preguntas muy variadas, pero se clasifican específicamente en dos en cuestionario de preguntas abiertas y cerradas.

En cuanto al análisis estadístico de los datos, según el tipo de investigación descriptiva, se hace necesario el empleo de estadística descriptiva, para construir y definir los parámetros de media de cada una de las variables, dimensiones, indicadores analizados.

Para Arias (2012), quien indica que aunque hay tendencia a generalizar a toda la población, las primeras conclusiones obtenidas tras un análisis descriptivo, es un estudio calculando una serie de medidas de tendencia central, para ver en qué medida los datos se agrupan o dispersan en torno a un valor central. Por su lado, Bavaresco (2008), señala que la estadística descriptiva registra los datos en tablas y los representa en gráficos. Calcula los parámetros estadísticos (medidas de centralización y de dispersión), que describen el conjunto estudiado.

\section{CONSIDERACIONES FINALES}

La modalidad en la transferencia de conocimiento y los mecanismos según las respuestas se encuentran ausentes. En cuanto a las formas de transferencia del conocimiento, se encuentran medianamente presente dentro del sector farmacéutico, significando que existe una debilidad en el proceso de conocimientos entre los grupos de trabajo. Los principios corporativos de las estrategias gerenciales se mostraron medianamente presente a pesar de que la interactividad e inspiración del recurso 
humano prevalecen en el desarrollo de los objetivos asignados. Se concluye que la primera variable (Transferencia de Conocimiento) se encuentra ausente y la segunda (Filosofía de Gestión) medianamente presente, comprometiendo así el éxito de la organización. En relación a lo anterior, señalan Rodríguez y Colina (2017) que "el éxito o fracaso de un proceso de gestión del conocimiento se vislumbra a la luz de los resultados obtenidos y sus repercusiones sociales" (pag. 11). Es así, que si no se evidencia en el éxito de la organización la transferencia de conocimiento, la organización debe revisarse gerencialmente.

Se recomienda a los directivos de las instituciones investigadas, establecer vínculos motivacionales con estas acciones emprendidas, teniendo la disposición de ánimo para la consecución de los fines pretendidos, y dar a conocer a los gerentes y empleados que participaron en la investigación, estos recursos; además del desarrollo de competencias del personal, permitiendo generar el conocimiento e integrarse la innovación cónsona con las nuevas tendencias gerenciales.

\section{REFERENCIAS CONSULTADAS}

1. Arias, F. (2012). El proyecto de Investigación. Introducción a la metodología científica. Caracas: Editorial Episteme.

2. Barroso, M. (2009). Meditaciones gerenciales. Venezuela. Editorial Galac.

3. Bavaresco (2008) Manual para la elaboración de Tesis, Monografías, Informes. Octava Edición. Editorial Rústico.

4. Briones, G.(1990) Métodos y técnicas avanzadas de investigación aplicadas a la educación y a las ciencias sociales. Bogotá: ICFES-PIIE. Cap. V: Análisis multivariado (con excepción de modelos logarítmicos lineales); cap. VI: Otras técnicas multivariadas.

5. Chávez, N. (2010). Introducción a la Investigación. Maracaibo: Ediluz.

6. Cohen, W., y Levinthal, D. (1990), "Fortune favours the prepared firm". Management Science, 40, pp. 227-251. 
conocimiento.

Extraído

de:

http://www.eumed.net/tesisdoctorales/2007/cavl/Transferencia\%20del\%20conoci $\underline{\text { miento.htm. }}$

7. Davis, K. y Newstron J. (2008). Comportamiento humano en el trabajo. Mc GrawHill. México.

8. Dawson, R. (2010). Developing Knowledge-based Client Relationships: The Future of Professional Services. Oxford: Ed. Butterworth Heinemann.

9. Vega, C. (2007). Integración de herramientas de tecnología de información "Portales colaborativos de trabajo" como soporte en la administración como soporte en la administración del conocimiento". Universidad Málaga

10. De las Salas, M., \& Bozo, R. (2014). Competencias gerenciales y desempeño laboral en empresas aseguradoras. CICAG, 12(2). Obtenido de http://publicaciones.urbe.edu/index.php/cicag/article/viewArticle/3558/4849

11. Dyer, J.H. y Nobeoka, K. (2000). Creating and managing a highperformance knowledge sharing network: The Toyota case. Strategic Management Journal, 21(3),345-367.

12. Gibson, J; Ivancevich, J; Donnelly, J y R, Konopaske (2015) Organizaciones: comportamiento, estructura procesos. Colección General. Mexico: Mc Graw Hill

13. Gonzales, M.L. (2011). La importancia de la motivación y las habilidades computacionales de los futuros profesores en el uso de las TIC. Revista Iberoamericana de Educación Superior (RIES), 2(3), 116-129. Recuperado de http://www.redalyc.org/

14. Hernández, R., Fernández, C., Baptista, P. (2014). Metodología de la Investigación. México: Editorial McGraw Hill.

15. Hurtado, J. (2010). El proyecto de investigación. Sypal. Caracas. Venezuela

16. Lam, P.L.; (1999), "The reification of absorptive capacity: a critical review and rejuvenation of the construct". Academy of Management Review, 31(4), pp.833863.

17. Nonaka,I., y Takeuchl,H. (1999): The knowledge-creating company: How japanese companies create the dynamics of innovation. Oxford University Press. 
18. Ordóñez P (2011), Investigaciones Europeas de Dirección y Economía de la Empresa Vol. 7, № 3. Universidad de Oviedo.

19. Palella y Martins (2006) Metodología de la Investigación Cuantitativa.3ra Edición. Editorial: Fedeupel pdf/2991/299124244006.pdf.

20. Reyes, J. (2011). Gestión por Competencia. Andersen Consulting, España.

21. Rodríguez Acasio, F., \& Colina Ysea, F. (2017). LA INVESTIGACIÓN DESDE LA GESTIÓN DEL CONOCIMIENTO EN EL CONTEXTO DE LAS UNIVERSIDADES NACIONALES EXPERIMENTALES. Revista Arbitrada Interdisciplinaria Koinonía, 1(1), 88-100. Recuperado de http://fundacionkoinonia.com.ve/ojs/index.php/revistakoinonia/article/view/17/10

22. Rodríguez, G (2009) Funciones y rasgos del liderazgo pedagógico en los centros de enseñanza Educación y Educadores, vol. 14, núm. 2, pp. 253-267. Universidad de La Sabana Cundinamarca, Colombia.

23. Serna, H (2006) Gerencia estratégica. Teoria - metodología- Alineamiento implementación y mapas estratégicos. Bogotá. Colombia. Disponible en: http://tangara.uis.edu.co/biblioweb/tesis/2015/156268.pdf

24. Testar, $X$ (2012), La transferencia de tecnologia y conocimiento universidadempresa en España: estado actual, retos y oportunidades. Colección Documentos CYD.

25. Von Krogh, G.; (2000): Facilitar la creación de conocimiento. Cómo desentrañar el misterio del co-nocimiento tácito y liberar el poder de la innovación, Oxford, Oxford Univer-sity Press.

\section{REFERENCES CONSULTED}

1. Arias, F. (2012). The research project. Introduction to scientific methodology. Caracas: Editorial Episteme.

2. Barroso, M. (2009). Management meditations. Venezuela. Editorial Galac.

3. Bavaresco (2008) Manual for the elaboration of Theses, Monographs, Reports. Eighth edition. Rustic Editorial. 
4. Briones, G. (1990) Advanced research methods and techniques applied to education and social sciences. Bogotá: ICFES-PIIE. chap. V: Multivariate analysis (with the exception of linear logarithmic models); chap. VI: Other multivariate techniques.

5. Chávez, N. (2010). Introduction to research. Maracaibo: Ediluz.

6. Cohen, W., and Levinthal, D. (1990), "Fortune favors the prepared firm". Management Science, 40, pp. 227-251.

7. knowledge. http://www.eumed.net/tesisdoctorales/2007/cavl/Transferencia\%20del\%20conoci miento.htm.

8. Davis, K. and Newstron J. (2008). Human behavior at work. Mc Graw-Hill. Mexico.

9. Dawson, R. (2010). Developing Knowledge-based Client Relationships: The Future of Professional Services. Oxford: Ed. Butterworth Heinemann.

10. Vega, C. (2007). Integration of information technology tools "Collaborative work portals" as support in the administration as support in the administration of knowledge." Universidad Málaga

11. De las Salas, M., \& Bozo, R. (2014). Managerial skills and work performance in insurance companies. CICAG, 12 (2). Retrieved from http://publicaciones.urbe.edu/index.php/cicag/article/viewArticle/3558/4849

12. Dyer, J.H. and Nobeoka, K. (2000). Creating and managing a highperformance knowledge sharing network: The Toyota case. Strategic Management Journal, 21 (3), 345-367.

13. Gibson, J; Ivancevich, J; Donnelly, J and R, Konopaske (2015) Organizations: behavior, structure processes. General Collection. Mexico: Mc Graw Hill

14. Gonzales, M.L. (2011). The importance of motivation and computational skills of future teachers in the use of ICT. Iberoamerican Journal of Higher Education (RIES), 2 (3), 116-129. Retrieved from http://www.redalyc.org/

15. Hernández, R., Fernández, C., Baptista, P. (2014). Investigation methodology. Mexico: McGraw Hill Publishing House.

16. Hurtado, J. (2010). The research project. Sypal. Caracas. Venezuela 
17.Lam, P.L .; (1999), "The reification of absorptive capacity: a critical review and rejuvenation of the construct". Academy of Management Review, 31 (4), pp.833863.

18. Nonaka, I., And Takeuchl, H. (1999): The knowledge-creating company: How to create companies in the dynamics of innovation. Oxford University Press.

19. Ordóñez P (2011), European Research on Business Management and Economics Vol. 7, No. 3. University of Oviedo.

20. Palella and Martins (2006) Quantitative Research Methodology 3rd Edition. 20. Editorial: Fedeupel pdf / 2991 / 299124244006.pdf.

21. Reyes, J. (2011). Management by Competition. Andersen Consulting, Spain.

22. Rodríguez Acasio, F., \& Col. Ysea, F. (2017). RESEARCH FROM THE MANAGEMENT OF KNOWLEDGE IN THE CONTEXT OF EXPERIMENTAL NATIONAL UNIVERSITIES. Journal Interdisciplinary Arbitrated Koinonía, 1 (1), 88-100.

Retrieved

from http://fundacionkoinonia.com.ve/ojs/index.php/revistakoinonia/article/view/17/10

23. Rodríguez, G (2009) Functions and features of pedagogical leadership in Education and Educators, vol. 14, no. 2, pp. 253-267. University of La Sabana Cundinamarca, Colombia.

24. Serna, H (2006) Strategic management. Theory - methodology - Alignment implementation and strategic maps. Bogotá Colombia. Available at: http://tangara.uis.edu.co/biblioweb/tesis/2015/156268.pdf

25. Testar, $X$ (2012), The transfer of technology and knowledge university-business in Spain: current status, challenges and opportunities. Documents Collection CYD.

26. Von Krogh, G .; (2000): Facilitate the creation of knowledge. How to unravel the mystery of tacit knowledge and unleash the power of innovation, Oxford, Oxford Univer- sity Press. 